\title{
Microhardness studies on some alum crystals
}

\author{
G SANGAIAH* and K KISHAN RAO \\ Department of Physics, Kakatiya University, Warangal 506009 , India \\ *Government College of Arts and Science, Kama Reddy 503111 , India \\ MS received 8 January 1993; revised 12 July 1993
}

\begin{abstract}
Microhardness studies were carried out using four alums. The hardness of these crystals was found to be more than alkali halides but was comparable with alkaline earth nitrates. The load variation of hardness has been discussed. Hardness results on these alums have been analysed taking into account the structure. It was observed that hardness varied with $a^{-4}$, where $a$ is the lattice constant. Preliminary results on dislocation etching are reported.
\end{abstract}

Keywords. Microhardness; dislocations; alums.

\section{Introduction}

Considerable amount of work has been reported on the growth of alum crystals (Buckley 1951; Holden and Singer 1968). Among them, potash alum has attracted the attention of many workers. The alums have the general formula $\mathrm{A}^{\mathrm{I}} \mathrm{B}^{\mathrm{III}}\left(\mathrm{SO}_{4}\right)_{2} 12 \mathrm{H}_{2} \mathrm{O}$. Though all the alums are cubic, they have three slightly different structures $(\alpha, \beta, \gamma)$. Data on several physical properties like densities, lattice constants, elastic constants, thermal expansion and dielectric constant for nearly 60 alums have been reviewed by Haussuhl (1961). Surprisingly studies are lacking on hardness which is an important strength property. Hence, it is planned to make a systematic study of hardness on some of the alums.

\section{Experimental}

Good quality single crystals of four alums, viz. potassium aluminium sulphate (KAS), ammonium aluminium sulphate (AAS), ammonium ferric sulphate (AFS) and potassium chromium sulphate $(\mathrm{KCrS})$, have been grown from their aqueous solutions by slow evaporation at a constant temperature around $35^{\circ} \mathrm{C}$. All the crystals were grown either by keeping the seed crystal on the base of the jar or by hanging the seed in the solution. Crystals of size 8 to $10 \mathrm{~mm}$ along one edge were obtained in a period of ten days. Chemical etching technique was employed to reveal dislocations in these crystals. Microhardness measurements were made using Leitz-Wetzlar hardness tester fixed with a Vicker's diamond pyramidal indenter. Hardness values are calculated from the expression

$$
H=1 \cdot 854\left(P / d^{2}\right),
$$

where $P$ is the load applied in $g$ and $d$ the diagonal length in $\mu$. 


\section{Results and discussion}

\subsection{Chemical etching}

For an initial assessment of the quality of crystals, chemical etchants have been developed to reveal dislocations in these crystals. KAS and AAS are highly transparent whereas AFS and KCrS crystals are pale yellow and dark blue respectively. Figure 1 shows typical growing of AAS crystal in the solution; the developing [111] faces can be seen in the photograph. Most of the crystals are bounded by [111] faces, occasionally some small [100] faces are also observed. The present studies are confined to wellgrown [111] faces only.

Chemical etching technique has been employed to study dislocations in these crystals. These studies not only help in studying the distribution of dislocations but sometimes provide information on the nature of growth also. Van Enckevort and Van der Linden (1979) successfully employed this method to show that KAS grown by them is due to screw dislocation mechanism. In the present study a number of etchants were tried but good etching action was revealed by $5 \%$ undersaturated solutions of the respective alums. The etching time is about $6 \mathrm{sec}$. The etch pits are more or less equilateral triangles; the shape is in conformity with the three-fold rotational symmetry of the crystal face. Figure 2 shows the alternate etch, polish and etch photographs on AAS. From these photographs it can be noticed that all the pits of figure $2 a$ persist in figure $2 b$, indicating that the pits are formed at the sites of dislocations only. The density of dislocations was found to be about $4 \times 10^{4} / \mathrm{cm}^{2}$ in these crystals. The distribution of pits is more or less uniform in all these crystals.

\subsection{Hardness}

The hardness of crystals is an important strength property. There is considerable work on alkali halides (Chin et al 1972; Pratap and Haribabu 1980) and semiconducting

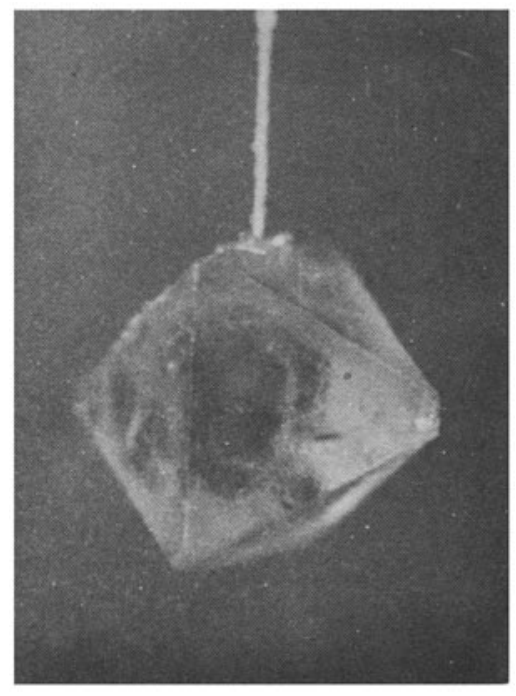

Figure 1. Growing crystal of AAS ( $\times 1$ ). 

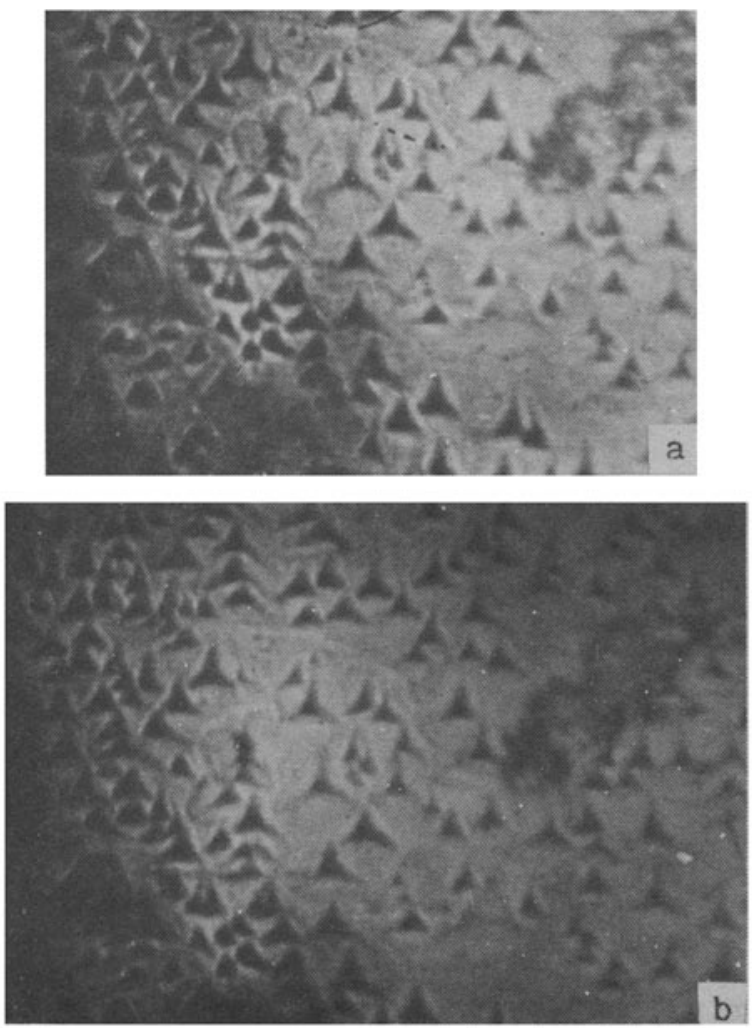

Figure 2. Etch pattern on (a) (111) surface, $\times 100$ and (b) etched after polishing the same crystal surface $(\times 100)$.

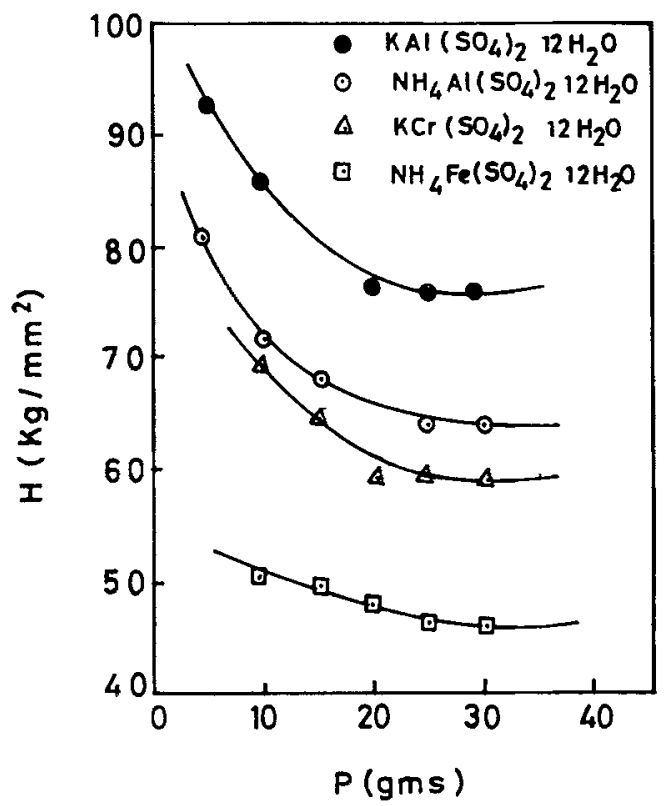

Figure 3. Plot of microhardness $(H)$ against load $(P)$. 
materials (Gilman 1973). Figure 3 shows the load variation of hardness for the four alums. It can be seen from the plots that hardness is large at low loads and decreases with increasing load. Above a load of $20 \mathrm{~g}$ the hardness becomes load independent in all the crystals. The present load variation results are similar to the results obtained on $\mathrm{LiF}$ (Upit and Varchanya 1973), on $\mathrm{KCl}$ and $\mathrm{NH}_{4} \mathrm{Cl}$ (Pratap and Haribabu 1980), and on rubidium halides (Thirmal Rao and Sirdeshmukh 1991). This type of load variation can be interpreted by using Kick's relation (Kick and Das 1885),

$$
P=k d^{n}
$$

where $k$ is a constant and $n$ the Meyer index; $n$ is expected to be 2, but most of the experimental data available show that $n$ is always less than 2. Hays and Kendall (1973) pointed out that load variation of the type observed in the present work can be interpreted by assuming the existence of Newtonian resultant pressure due to the specimen itself.

In the present work a slightly different approach has been made to analyse the load variation results. Figure 4a shows the plots between $P$ and $d^{2}$; it can be seen that the plots do not pass through the origin but make intercepts on load axis. The intercepts $(w)$ could be due to loading error or due to the resistance offered by the samples to the load. Now a series of new plots are drawn between $(P-w)$ and $d^{2}$. Now these plots pass through the origin as shown in figure $4 \mathrm{~b}$. The value of $w$ is not the same for all the crystals but varies from $1.25 \mathrm{~g}$ to $3.5 \mathrm{~g}$ as shown in table 1 . Hence it appears reasonable to attribute $w$ to the resistance offered by the crystals for plastic
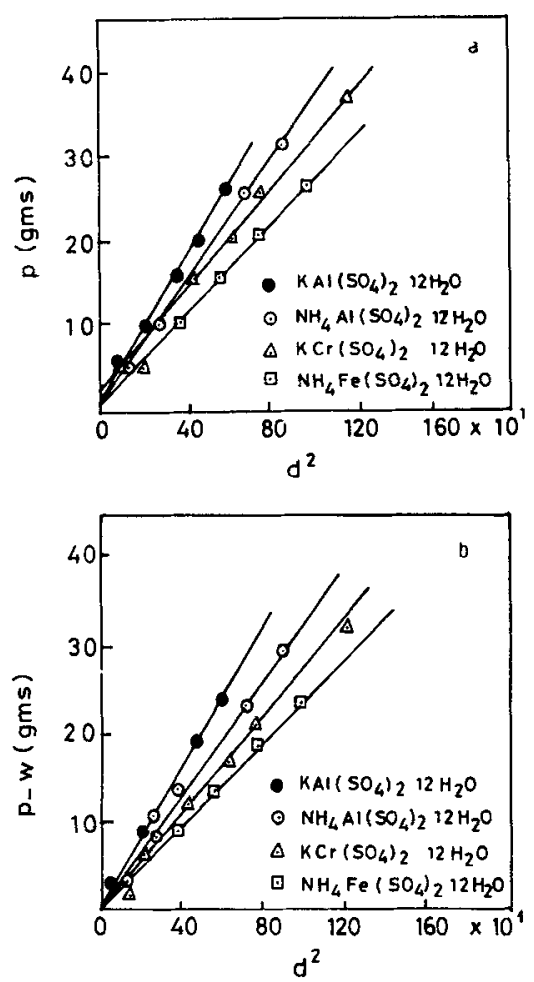

Figure 4. (a) Plot of load (P) against square of diagonal length $\left(d^{2}\right)$, and (b) plot of $(P-w)$ against square of diagonal length $\left(d^{2}\right)$. 
Table 1. Hardness parameters for some alums.

\begin{tabular}{lccccc}
\hline Crystal & $H\left(\mathrm{~kg} / \mathrm{mm}^{2}\right)$ & $w(\mathrm{~g})$ & $\begin{array}{c}H\left(\mathrm{~kg} / \mathrm{mm}^{2}\right) \\
(\text { corrected })\end{array}$ & $a(\AA)$ & $\begin{array}{l}\left(4 / a^{4}\right) \\
\left(\AA^{-4}\right)\end{array}$ \\
\hline KAS & 76.6 & 1.5 & 72.8 & 12.158 & 0.01172 \\
KCrS & 59.5 & 3.5 & 52.6 & 12.197 & 0.01157 \\
AAS & 63.5 & 2.0 & 59.2 & 12.240 & 0.01140 \\
AFS & 46.7 & 1.5 & 41.2 & 12.322 & 0.01110 \\
\hline
\end{tabular}

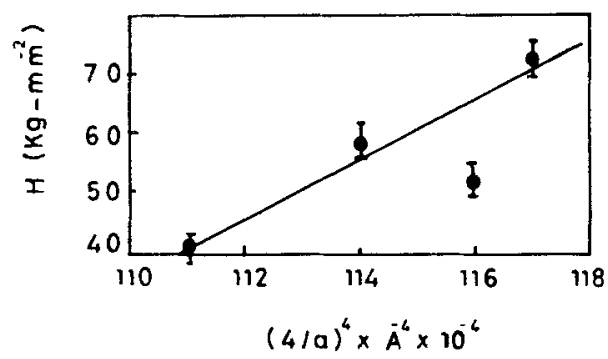

Figure 5. Plot of corrected hardness $(H)$ against $a^{-4}$.

deformation rather than to the loading error. The corrected hardness is then obtained by substituting $(P-w)$ for $P$ in (1). The values of $H$ thus obtained are given in table 1 .

Although hardness has been defined in several ways, Gilman (1973) treats it as a strength microprobe. He correlated the hardness with other strength-dependent parameters like lattice constant, bulk modulus, etc. for III-V compounds. It can be seen from table 1 that hardness varies from 41.2 to $72.8 \mathrm{~kg} / \mathrm{mm}^{2}$ and on Mohs scale in the range 2-3. These crystals are harder than monovalent alkali halides, but are softer than the divalent alkaline earth fluorides, and are comparable with alkaline earth nitrates. Haussuhl (1961) analysed the elastic constants of ionic crystals and has shown that the elastic constants scale with the parameter

$$
(z e)^{2} / r^{4}
$$

where $z$ is the valency, $e$ the electronic charge, and $r$ the interionic distance. Bragg (1967) described the structure of alums as follows: $\mathrm{R}^{+}$and $\mathrm{R}^{++}$have a rock-salt arrangement, there are alternate [111] planes of $\mathrm{R}^{+}$and $\mathrm{R}^{+++}$, with a complex mass of $\mathrm{SO}_{4}$ and $\mathrm{H}_{2} \mathrm{O}$ lying between them. It is clear that neither the structure nor bonding in the alums is as simple as in the alkali halides. Yet, Haussuhl (1961) extends the same analysis to the alums and shows that the elastic constants scale with the parameter $(z e)^{2} / r^{4} ;$ he used $z=2$ and $r=a / 4$, where $a$ is lattice constant. Since hardness is a mechanical property akin to the elastic moduli, it may be expected to show a similar behaviour. In figure 5 the corrected hardness is plotted against $(4 / a)^{4}$; a linear plot is obtained though with some scattering. The hardness and elastic constants of alums are together consistent with an ionic model.

\section{Acknowledgements}

The authors are grateful to Prof. D B Sirdeshmukh for useful discussions and suggestions and to the referees for numerous suggestions. 


\section{References}

Bragg W L 1967 Crystalline state (London: G Bell \& Sons Ltd) Vol. 1, p. 225

Buckley H E 1951 Crystal growth (New York: Wiley)

Chin G Y, Van Utert L G, Green M L and Zydzik G J 1972 Scr. Metall. 6503

Gilman J J 1973 The science of hardness testing and its research applications (Ohio: Amer. Soc. Metals)

Haussuhl S $1961 Z$. fur Kristall 11624

Hays C and Kendall E G 1973 Metallography 6275

Holden A and Singer P 1968 Crystals and crystal growing (Bombay: Vakil's, Febber \& Simons)

Kick $\mathrm{F}$ and Das Gesetzder 1885 Proportionalen winderstande and science anwendung (ed) Leipzit Felix

Pratap K J and Haribabu V 1980 Bull. Mater. Sci. 243

Thirmal Rao T and Sirdeshmukh D B 1991 Cryst. Res. Technol. 26 K53

Upit G P and Varchanya S A 1973 The science of hardness testing and its research applications (Ohio: Amer. Soc. Metals)

Van Enckevort W J P and Van der Linden W H 1979 J. Cryst. Growth 47196 